\title{
Hundred Thousand per High Powered
}

Field

National Cancer Institute

\section{Source}

National Cancer Institute. Hundred Thousand per High Powered Field. NCI Thesaurus.

Code C98743.

A unit of measurement equal to one hundred thousand entities per unit of area equal to one high powered field. 